\title{
Modification of a Weighting Function for NLFM Radar Signal Designing
}

\author{
C. LEŚNIK* AND A. KAWALEC \\ Military University of Technology, Gen. S. Kaliskiego 2, 00-908 Warszawa, Poland \\ Modification of the weighting raised cosine with a power $n$ function by \\ time convolution with auxiliary changeable time duration uniform rectangu- \\ lar window is presented in this paper. The analytical form of the modified \\ weighting raised cosine function for fixed power in frequency domain was \\ derived. Proposed method was applied to fine tuning of properties of the \\ nonlinear frequency modulation of radar signals. Synthesis of nonlinear fre- \\ quency modulation radar signals problem was solved using the well-known \\ principle of stationary phase. The selected testing results are shown and \\ some final conclusions are formulated.
}

PACS numbers: 84.40.Ua, 84.40.Xb

\section{Introduction}

It is well known that the synthesis of radar signals, influences much to the radar detection problem of targets. Concerning the matched filter in receiver only, it maximizes the peak signal to mean noise power ratio, which gives maximum range of targets detection. The optimal filter characteristic depends on the wave form.

For the modern radar applied with a complex signal an output signal of the matched filter has a shape of a short signal in time. It is a particular interest for requirement of target discrimination in distance. However, in the particular case there are side-lobes, which can mask weak echoes of targets. This phenomenon, rarely existing in practice, can be improved by applying convenient weighting function in order to modify a transmittance of the matched filter. This operation causes mismatching, which reduces a signal to noise ratio and decreases a range of detection of targets.

Another unlosses technique, which decreases a side-lobe level is application of a complex signal with a nonlinear frequency modulation. In this operation usually stationary phase method can be applied and weighting functions are useful as well.

*corresponding author; e-mail: Czeslaw.Lesnik@wel.wat.edu.pl 
It is known that a searching into weighting functions, having specific properties, is a very important problem in the spectral analysis of signals.

Known methods are available in synthesis of weighting functions, well described in literature, for example time convolution of parent windows [1], multiple time convolution of rectangular windows [2, 3] or multiple time auto and cross convolution of other well known windows $[4,5]$. These so-called convoluted windows have greater side-lobe attenuation and fast decay. Unfortunately, width of the main lobe is increased significantly usually.

The fine tuning of property of raised cosine with power $n$ function by its time convolution with the rectangular window having changeable time duration is proposed in this paper.

\section{Design of a weighting function}

Generally a finite time duration weighting function $w_{T}(t)$ can be expressed as $w_{T}(t)=w(t) r_{T}(t)$,

where $w(t)$ denotes an infinite time duration considering function, $r_{T}(t)$ denotes uniform rectangular window (rect in short)

$$
r_{T}(t)=\operatorname{rect}\left(\frac{t}{T}\right)=\left\{\begin{array}{ll}
1 & \text { for }|t| \leq \frac{T}{2}, \\
0 & \text { for elsewhere }
\end{array} \quad T>0\right.
$$

where $T$ denotes window duration.

In frequency domain Eq. (1) is given by

$$
W_{T}(\omega)=\frac{1}{2 \pi}\left[W(\omega) * R_{T}(\omega)\right]
$$

where $W(\omega)$ and $R_{T}(\omega)$ are the Fourier transforms of $w(t)$ and $r_{T}(t)$, suitably, and * denotes the convolution operator. A method of a weighting function properties fine-tuning for nonlinear frequency modulation (NLFM) radar and sonar signals designing is proposed here. It was based on the time convolution of the $w_{T}(t)$ window function and the auxiliary changeable time duration uniform rectangular window $r_{\tau}(t)$ :

$$
w_{\tau}(t)=w_{T}(t) * r_{\tau}(t)=\left[w(t) r_{T}(t)\right] * r_{\tau}(t),
$$

where $w_{\tau}(t)$ denotes modified weighting function. The $r_{\tau}(t)$ is defined as follows:

$$
r_{\tau}(t)=\operatorname{rect}\left(\frac{t}{\tau}\right)=\left\{\begin{array}{ll}
\frac{1}{\tau} & \text { for }|t| \leq \frac{\tau}{2}, \\
0 & \text { for elsewhere, }
\end{array} \quad \tau>0,\right.
$$

where $\tau$ denotes window changeable time duration. In frequency domain Eq. (3) is given by

$$
W_{\tau}(\omega)=\frac{1}{2 \pi}\left[W(\omega) * R_{T}(\omega)\right] R_{\tau}(\omega),
$$

where $W_{\tau}(\omega)$ and $R_{\tau}(\omega)$ are the Fourier transforms of $w_{\tau}(t)$ and $r_{\tau}(t)$, suitably.

After convolution the resultant window $w_{\tau}(t)$ will have the time duration equal to $(T+\tau)$. So the time change scale factor is 


$$
a=\frac{T}{T+\tau} .
$$

Taking advantage of the Fourier transform (the scaling property)

$$
x\left(\frac{t}{a}\right) \leftrightarrow|a| X(a \omega)
$$

general form of the modified weighting function (5) can be drawn

$$
W_{\tau}(\omega)=\frac{T}{2 \pi(T+\tau)}\left[W\left(\frac{T}{T+\tau} \omega\right) * R_{T}\left(\frac{T}{T+\tau} \omega\right)\right] R_{\tau}\left(\frac{T}{T+\tau} \omega\right) .
$$

For example, let us consider in the time domain a well-known [1] weighting raised cosine with a power $n$ function described by

$$
w_{T}(t)=w(t) r_{T}(t)=\left[k+(1-k) \cos ^{n}\left(\pi \frac{t}{T}\right)\right] \operatorname{rect}\left(\frac{t}{T}\right),
$$

where $k$ denotes real value parameter, $k \in\langle 0,1\rangle, n$ denotes integer value parameter, $n \geq 1, T$ denotes pulse duration.

This function has of great importance in pulse-compression signals with NLFM both in a radar and a sonar [6-8].

Using the method described above for $n=2$, expression (8) can be drawn

$$
\begin{aligned}
w_{T}(t) & =\left[k+(1-k) \cos ^{2}\left(\pi \frac{t}{T}\right)\right] \operatorname{rect}\left(\frac{t}{T}\right) \\
= & {\left[\frac{k+1}{2}+\frac{(1-k)}{2} \cos \left(2 \pi \frac{t}{T}\right)\right] \operatorname{rect}\left(\frac{t}{T}\right) . }
\end{aligned}
$$

Therefore, in frequency domain we can obtain

$$
\begin{aligned}
& W(\omega)=(k+1) \pi \delta(\omega)+\frac{1-k}{2} \pi\left[\delta\left(\omega-\frac{2 \pi}{T}\right)+\delta\left(\omega+\frac{2 \pi}{T}\right)\right] \\
& R_{T}(\omega)=T \operatorname{Sa}\left(\omega \frac{T}{2}\right), \quad \text { and } \\
& W_{T}(\omega)=\frac{1}{2 \pi}\left[W(\omega) * R_{T}(\omega)\right] \\
& =\left\{\frac{k+1}{2} \delta(\omega)+\frac{1-k}{4}\left[\delta\left(\omega-\frac{2 \pi}{T}\right)+\delta\left(\omega+\frac{2 \pi}{T}\right)\right]\right\} * T \operatorname{Sa}\left(\omega \frac{T}{2}\right)
\end{aligned}
$$

where $S a($.$) denotes a function in a shape of S a(x)=\frac{\sin (x)}{x}$. Taking advantage of the delta distribution expression, (10) can be rearranged to a form

$$
\begin{gathered}
W_{T}(\omega)=\frac{(k+1)}{2} T \mathrm{Sa}\left(\omega \frac{T}{2}\right)+\frac{1-k}{4} T \mathrm{Sa}\left[\left(\omega-\frac{2 \pi}{T}\right) \frac{T}{2}\right] \\
+\frac{1-k}{4} T \mathrm{Sa}\left[\left(\omega+\frac{2 \pi}{T}\right) \frac{T}{2}\right] .
\end{gathered}
$$

The auxiliary changeable time duration window 


$$
r_{\tau}(t)=\frac{1}{\tau} \operatorname{rect}\left(\frac{t}{\tau}\right)
$$

has a Fourier transform

$$
R_{\tau}(\omega)=\operatorname{Sa}\left(\omega \frac{\tau}{2}\right) .
$$

Therefore, the modified weighting function (11) in time and frequency domain can be drawn suitably

$$
\begin{aligned}
w_{\tau}(t) & =w_{T}(t) * r_{\tau}(t) \\
= & \left\{\left[\frac{k+1}{2}+\frac{(1-k)}{2} \cos \left(2 \pi \frac{t}{T}\right)\right] \operatorname{rect}\left(\frac{t}{T}\right)\right\} *\left[\frac{1}{\tau} \operatorname{rect}\left(\frac{t}{\tau}\right)\right], \\
W_{\tau}(\omega) & =W_{T}(\omega) R_{\tau}(\omega) \\
= & \left\{\frac{(k+1)}{2} T \operatorname{Sa}\left(\omega \frac{T}{2}\right)+\frac{1-k}{4} T \operatorname{Sa}\left[\left(\omega-\frac{2 \pi}{T}\right) \frac{T}{2}\right]\right. \\
+ & \left.\frac{1-k}{4} T \mathrm{Sa}\left[\left(\omega+\frac{2 \pi}{T}\right) \frac{T}{2}\right]\right\} \mathrm{Sa}\left(\omega \frac{\tau}{2}\right) .
\end{aligned}
$$

After time scaling by factor $a=T /(T+\tau)$ expression (12) can be rearranged to a form

$$
\begin{aligned}
& W_{\tau}(\omega)= \\
& =\left\{\frac{(k+1)}{2} T \operatorname{Sa}\left(\omega \frac{T}{2} \frac{T}{T+\tau}\right)+\frac{1-k}{4} T \operatorname{Sa}\left[\left(\omega-\frac{2 \pi}{T} \frac{T+\tau}{T}\right) \frac{T}{2} \frac{T}{T+\tau}\right]\right. \\
& \left.+\frac{1-k}{4} T \operatorname{Sa}\left[\left(\omega+\frac{2 \pi}{T} \frac{T+\tau}{T}\right) \frac{T}{2} \frac{T}{T+\tau}\right]\right\} \operatorname{Sa}\left(\omega \frac{\tau}{2} \frac{T}{T+\tau}\right) \frac{T}{T+\tau} .
\end{aligned}
$$

The synthesis of a weighting function for the other power of $n$ in expression (8) will be done in the same way.

Equation (13) has been determined as a base for researching property of a weighting function and for a synthesis of a radar signal with an NLFM.

\section{Testing results}

The simulation model including the weighting functions generator and radar signals generator has been developed. It was done in order to evaluate the time duration changes of the auxiliary uniform rectangular window on the property of the weighting functions and radar pulse signals. As a result, a series of characteristics have been obtained. Some selected from all are presented here.

Time convolution of the weighting raised cosine with a power $n$ function and auxiliary changeable time duration uniform rectangular window has been done 
numerically. Normalized magnitude spectrum of the modified windows for different values of the parameter $n$, from $n=1$ to $n=6$, versus normalized frequency are depicted in Fig. 1. The values of $k$ and $\tau$ parameters have been obtained by recursive finding of the minimum side-lobes level. The same characteristics for non-modified rectangular and raised cosine windows for comparison are depicted as well. Presented characteristics clearly show that modified windows side-lobes suppression improved from about $14 \mathrm{~dB}$ to $27 \mathrm{~dB}$ in comparison to non-modified windows has been achieved. It depends on parameter $n$ value. Unfortunately, the main lobe width at $-3 \mathrm{~dB}$ level increase from 1.28 to 1.08 times when $n$ changes from from $n=1$ to $n=6$. It is caused by a modification mentioned above.

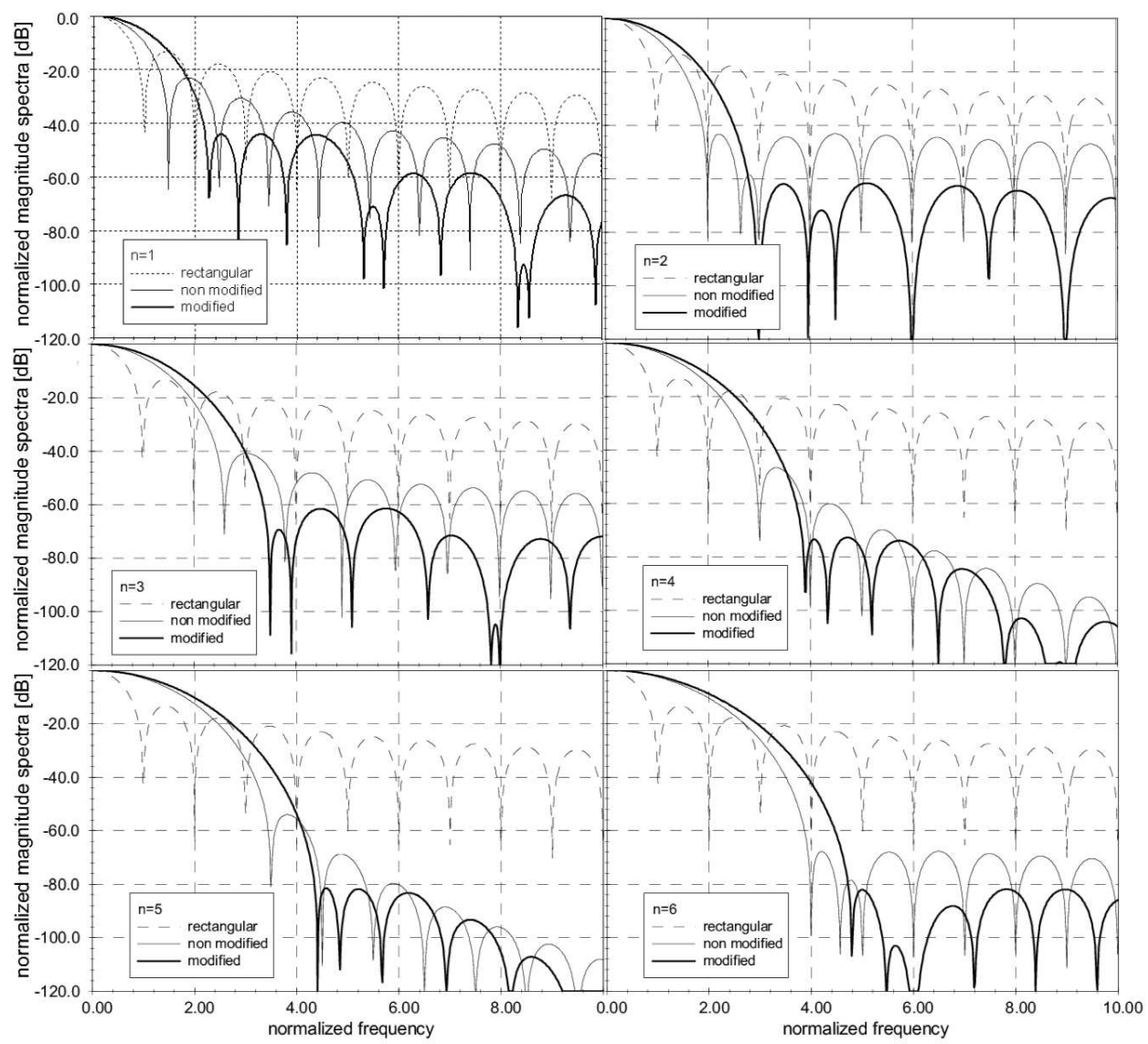

Fig. 1. Normalized magnitude spectrum of the modified, non-modified, and rectangular windows for different $n$ parameter values.

For example, we can obtain a comparable side-lobes level for the modified window with $n=2$ and the non-modified window with $n=6$, but second one has main lobe width at $-3 \mathrm{~dB}$ level 1.35 times wider. 
Design of the transmitted NLFM radar signal has been done in the next research stage. The matched filter output signal is given by the input filter autocorrelation function. The signal autocorrelation function is determined by the inverse Fourier transform of the energy spectral density. Therefore, in order to generate a NLFM signal with uniform amplitude we can use the signal with the magnitude spectrum described by a square root of the weighting function, raised cosine with a power $n$ for example. One can find the nonlinear frequency law by applying the stationary-phase concept as has been suggested by Fowle, Cook, and Bernfeld [6, 7].

The same method has been used in this paper. As a result, some characteristics have been obtained. Several examples are presented here. Normalized magnitude output of matched filter NLFM signals versus normalized to pulse duration time are depicted in Fig. 2 and Fig. 3. The characteristics are shown for the magnitude spectrum described by non-modified and modified weighting function and for two different values of the $B T$ product.

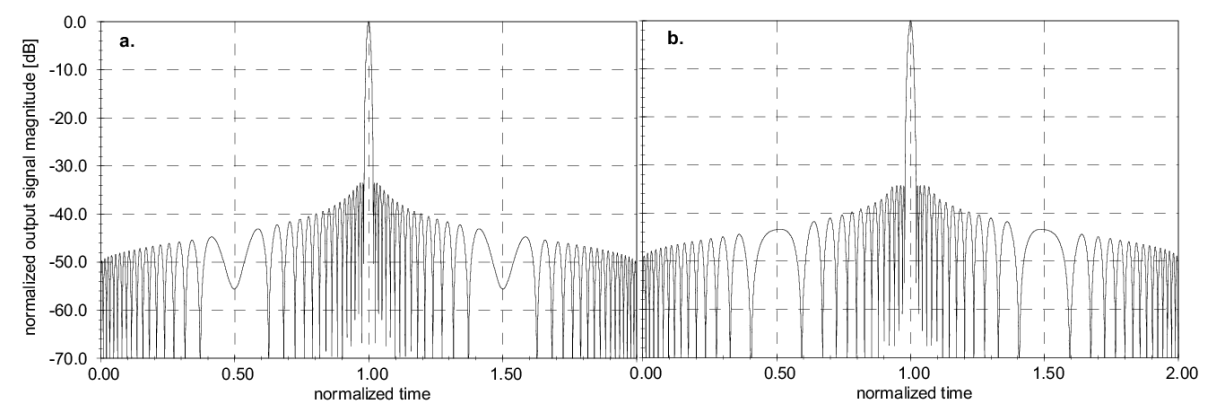

Fig. 2. Normalized magnitude output matched filter signal for the non-modified (a) $(k=0.113, \tau=0)$ and modified (b) $(k=0.2153, \tau=0.535 T)$ weighting function, and $B T=100$.

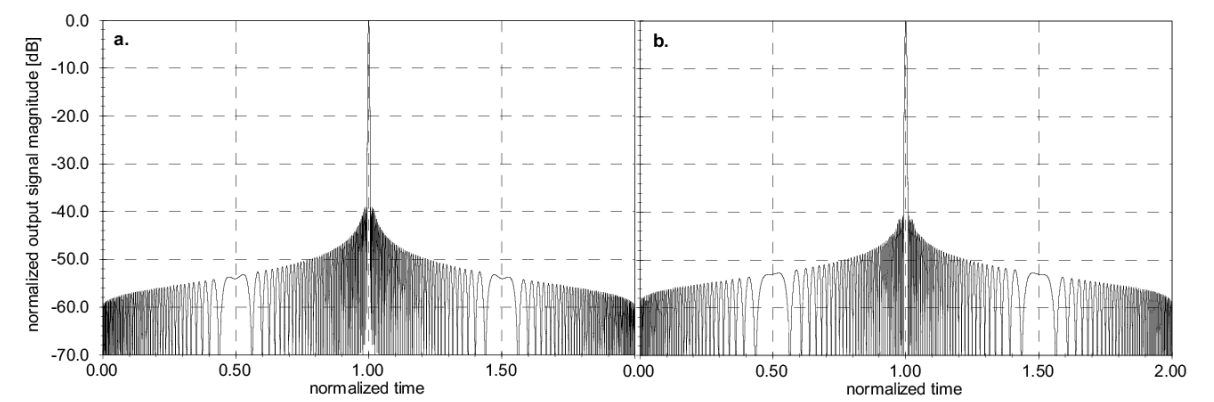

Fig. 3. Normalized magnitude output matched filter signal for the non-modified (a) $(k=0.09, \tau=0)$ and modified (b) $(k=0.094, \tau=0.415 T)$ weighting function, $B T=300$. 
As the results of the recursive finding of the minimum side-lobes level by values of the $k$ and $\tau$ parameters changing the $0.8 \mathrm{~dB}$ improvement of this level for $B T=100$ and $1.8 \mathrm{~dB}$ for $B T=300$ have been obtained.

\section{Summary}

The main aim of this paper is to show both weighting function properties fine tuning method and several initial results. The raised cosine with a power $n$ weighting function side-lobes suppression improvement from $14 \mathrm{~dB}$ to $27 \mathrm{~dB}$ has been achieved. It depends on a power $n$ value. The main lobe width has been increased simultaneously. The side-lobes suppression of the matched filter output NLFM radar signal has been improved as well, but far less. This depends on a signal $B T$ product.

The method, which has been proposed, can be extended to the different types of the weighting functions. Generalization of the method was presented and exact calculation of the modified windows parameters will be done in the next stage of the research in the future.

The method, proposed in this paper, can be applied for designing of radar signals. We can expect more applications of this method. For example, it can be applied for both the filter designing and harmonic analysis.

\section{Acknowledgments}

This work was supported by the Ministry of Science and Higher Education from sources for science in the years 2007-2010 under Commissioned Research Project PBZ-MNiSW-DBO-04/I/2007.

\section{References}

[1] F.J. Harris, Proc. IEEE 66, 51 (1978).

[2] P. Wen, IEEE Trans. Instrumentation Measurement 41, 403 (1992).

[3] X. Dai, R. Gretsch, IEEE Trans. Instrumentation Measurement 43, 204 (1994).

[4] I. Reljin, B. Reljin, V. Papić, P. Kostić, in: Proc. 9th Conf. MELECON, Tel Aviv (Israel) 1998, Vol. II, p. 878.

[5] I.S. Reljin, B.D. Reljin, V.D. Papić, IEEE Trans. Instrumentation Measurement 56, 1025 (2007).

[6] E.N. Fowle, IEEE Trans. Information Theory IT-10, 61 (1964).

[7] Ch.E. Cook, M. Bernfeld, Radar Signals. An Introduction to Theory and Application, Artech House, Inc., Boston 1993.

[8] N. Levanon, E. Mozeson, Radar Signals, Wiley, Hoboken, New Jersey 2004. 